\title{
Pregnancy and STD Prevention Counseling Using An Adaptation of Motivational Interviewing: A Randomized Controlled Trial
}

\begin{abstract}
CONTEXT: Given levels of unintended pregnancy and STDs, an effective counseling intervention is needed to improve women's consistent use of effective prevention methods.

METHODS: A sample of 764 women aged 16-44 who were at risk of unintended pregnancy were enrolled in a randomized controlled trial in North Carolina in 2003-2004. Intervention participants received pregnancy and STD prevention counseling, adapted from motivational interviewing, both at enrollment and two months later; controls received only a session of general health counseling. Levels of contraceptive use (categorized as high, low or none on the basis of the effectiveness of the method and the consistency of use) and barriers to use were measured at two, eight and 12 months; chi-square tests were used to compare selected outcomes between the groups. Rates of unintended pregnancy and chlamydia infection were assessed over the study period.
\end{abstract}

RESULTS: At baseline, 59\% of all participants reported a high level of contraceptive use, $19 \%$ a low level and $22 \%$ nonuse. At two months, the proportions of intervention and control participants who had improved their level of use or maintained a high level ( $72 \%$ and $66 \%$, respectively) were significantly larger than the proportions who had reported a high level of use at baseline (59\% and 58\%, respectively). No significant differences were found between the groups at 12 months, or between baseline and 12 months for either group. During the study, 10-11\% of intervention and control participants became pregnant, 1-2\% received a chlamydia diagnosis and 7-9\% had another STD diagnosed.

CONCLUSIONS: Repeated counseling sessions may be needed to improve contraceptive decision-making and to reduce the risk of unintended pregnancy and STDs.

Perspectives on Sexual and Reproductive Health, 2007, 39(1):21-28, doi: 10.1363/3902107

Women's health status in the United States is adversely affected by the occurrence of unintended pregnancies and STDs. Approximately half of all pregnancies in the United States are unintended at the time of conception, ${ }^{1,2}$ and these pregnancies are associated with higher rates of induced abortion ${ }^{2-4}$ and low-birth-weight infants, ${ }^{5}$ late initiation of prenatal care, ${ }^{6}$ use of harmful substances such as tobacco and alcohol during pregnancy, ${ }^{3}$ and increased risk of domestic violence. ${ }^{7}$ An estimated 19 million new STDs occur each year in the United States, and these infections put women at risk for long-term complications, including AIDS, pelvic inflammatory disease, ectopic pregnancy and infertility. ${ }^{9}$

More than half of all unintended pregnancies occur among the $7 \%$ of women who do not use any contraceptive method and yet do not want, or are not trying, to get pregnant. ${ }^{3}$ Women of all reproductive ages report contraceptive nonuse, ${ }^{3,10-13}$ and many women with unintended pregnancies also report inconsistent use, or use of an ineffective method. Nonuse and inconsistent use of condoms put these women at risk of contracting STDs.

Health care providers are in a unique position to counsel women about behaviors that place them at risk of unintended pregnancy and STDs. Prevention strategies should include attention to specific risk-taking behaviors and should emphasize targeted risk reduction counseling for all sexually active women. ${ }^{14,15}$ Such counseling should cover contraceptive use patterns (e.g., consistency of method use and effectiveness of method) and risk-taking behaviors (e.g., having multiple partners, having unprotected sex, having sex with partners who are at risk of HIV or other STDs). ${ }^{16}$ Contraceptive behaviors may be the risk factors that are most amenable to change through a health care visit. Consistent and correct use of contraceptives is the primary determinant of their effectiveness. ${ }^{17}$

Although health care providers can offer counseling on patterns of contraceptive use and risky behaviors, the opportunity is often missed. A minority of providers adequately counsel women on their contraceptive needs, and providers often fail to ask about pregnancy intention, sexual risk-taking behaviors, or the need for pregnancy or disease prevention. ${ }^{18-20}$ Providers have described several barriers to giving reproductive health counseling that are similar to barriers to offering general preventive health counseling-time restraints, inadequate reimbursement, lack of perceived need (by both clients and providers) and lack of training to address sensitive reproductive health issues. $^{21-25}$

No standardized intervention in routine clinical care settings has been proven effective in changing behaviors
By Ruth Petersen, Jennifer Albright, Joanne M. Garrett and Kathryn M. Curtis

Ruth Petersen is associate professor, Department of Maternal and Child Health, School of Public Health; Jennifer Albright is project manager, Center for Women's Health Research; and Joanne M. Garrett is professor, Department of Obstetrics and Gynecology, School of Medicine-all at University of North Carolina, Chapel Hill. Kathryn M. Curtis is epidemiologist, Division of Reproductive Health, Centers for Disease Control and Prevention, Atlanta. 
that might lead to a decrease in unintended pregnancies and STDs. ${ }^{26}$ Although a number of interventions have targeted HIV and STD prevention in both domestic and international settings, most have not considered a woman's pregnancy intentions in counseling or effectively incorporated behavioral theory to address sexual risktaking behaviors. The few interventions that have been informed by successes in other areas of health promotion have generally been implemented for specific high-risk clients outside of clinical settings (e.g., individuals in drug treatment, ${ }^{27}$ those in high-risk communities ${ }^{28}$ or incarcerated individuals $\left.{ }^{29}\right)$. The structure of some of these interventions (e.g., ranging from three four-hour sessions to five 90-minute sessions ${ }^{27,30-32}$ ) limits the ease with which they can be incorporated into standard clinical care.

One advance in behavioral counseling that providers can use to address the complexities of pregnancy and STD prevention counseling is motivational interviewing. ${ }^{33-35}$ This technique emphasizes the development of the client's self-efficacy ${ }^{36}$ with an appreciation for the different stages that a client may go through in adopting a health behavior, ${ }^{37}$ such as consistent use of an effective contraceptive. Motivational interviewing, which was developed by William Miller through his work in the 1970s and 1980s with problem drinkers, ${ }^{33}$ can be used to help clients recognize and potentially change behaviors that put their health at risk. The approach stresses empathy and reflective listening, while identifying discrepancies between behaviors (e.g., inconsistent contraceptive use) and broad goals (e.g., avoiding an unintended pregnancy). ${ }^{33,35}$

To address the lack of a standardized and proven counseling intervention in clinical settings, we evaluated the effectiveness of a behavioral-based intervention designed to reduce the risk and occurrence of unintended pregnancy and STDs among women at risk of unintended pregnancy.

\section{METHODS}

\section{Study Design}

This study was conducted at three primary care facilities affiliated with the Department of Family Medicine of the University of North Carolina at Chapel Hill. These facilities serve clients of all ages, including more than 6,000 women of reproductive age, from numerous counties across central North Carolina. At the beginning of this study, $66 \%$ of female clients of reproductive age were white, $27 \%$ were black and $6 \%$ were Asian, Hispanic or American Indian. Approximately 10\% paid for their care, and $19 \%$ were covered by Medicaid.

To be eligible for enrollment in the study, women visiting the clinics between March 2003 and September 2004 had to be 16-44 years old, at risk of unintended pregnancy (i.e., they were not pregnant and not planning a pregnancy within a year, they were not using an IUD, and neither they nor their partners were sterilized) and interested in participating. The study was described to potential participants as a "new information and counseling program about reproductive health for women who are NOT trying to get pregnant." Prospective participants gave written consent and provided a urine sample for pregnancy testing and DNA probe testing for chlamydia.

In this randomized controlled trial, the intervention group received pregnancy and STD prevention counseling with a health educator at enrollment and two months later in a booster session. The control group received brief, general counseling at enrollment on preventive health care (e.g., smoking, diet, exercise) that intentionally excluded counseling on pregnancy and STD prevention; these women received no further counseling. We used a random-numbers table to generate group assignments in permuted block sizes of 100, and placed sealed envelopes with the assignments at each recruitment site. Envelopes were opened after participants were screened for eligibility, gave informed consent and tested negative for being pregnant.

Participants completed self-administered baseline questionnaires, which included questions on general preventive health (e.g., smoking, exercise), contraceptive use, perceived barriers to use, level of pregnancy intention (i.e., wants to get pregnant now, wants to get pregnant in the near future, does not want to be pregnant in the near future, does not want to be pregnant, does not know) and occurrence of recent pregnancies and STDs. These questionnaires had been developed and pilot-tested in a population of women similar to the study population, using cognitive-response interviews, a qualitative evaluation method. $^{38}$

Participants completed follow-up questionnaires two, eight and 12 months after enrollment. At the 12-month follow-up, urine samples were collected for pregnancy and chlamydia testing; after this follow-up, participants' medical records were reviewed to assess their use of reproductive health care services and counseling during the study period. Women in both groups were compensated $\$ 25$ at the end of their participation. The study was completed in September 2005. The biomedical institutional review board of the University of North Carolina at Chapel Hill approved the study protocol.

\section{Women's Reproductive Assessment Program}

The intervention-the Women's Reproductive Assessment Program (WRAP)-was delivered by experienced health educators associated with and trained for this project. It used a counseling model adapted from motivational interviewing, ${ }^{39}$ following such principles as expressing empathy and supporting self-efficacy. ${ }^{33}$ This model emphasized three elements: exploring discrepancies between pregnancy intention and contraceptive use, and between STD risk and condom use; sharing information with participants; and promoting behaviors to reduce risk. ${ }^{35}$ To standardize the counseling, we provided educators with 30-40 hours of training on contraceptives, 
pregnancy and STD prevention counseling, motivational interviewing, clinic operation, study design and implementation, and the basics of smoking cessation, exercise and nutrition counseling. Quality control measures (e.g., random observation of counseling sessions, feedback from the project manager) were used throughout the study period.

The focus of the initial WRAP session was to encourage women to adopt consistent, effective contraceptive use, and condom use for the prevention of STD, including HIV, infection. Health educators elicited information about participants' perceived barriers to consistent, effective contraceptive use (e.g., by asking what kinds of situations made it hard to use a contraceptive method) and their level of self-efficacy and motivation for adopting risk reduction behaviors (e.g., by exploring how sure they were that they would use a contraceptive in the next 30 days). Educators used participants' baseline responses to evaluate pregnancy intention, contraceptive use patterns and high-risk sexual behaviors, and to individualize their counseling on contraceptive use and risk reduction strategies.

For women who were already consistently using contraceptives for pregnancy prevention and condoms for disease prevention, a risk reduction step might simply be continuing current practice. For women with low selfefficacy or those in an early stage of adopting consistent and effective contraceptive use, ${ }^{33}$ risk reduction steps might involve cognitive issues, such as improving communication with partners. The initial session also offered women the opportunity to obtain, or receive a referral for, any type of contraceptive. If intervention participants elected to start or change contraceptive methods, their primary care provider was notified and the information was added to their medical record.

Two months after the enrollment session, WRAP health educators conducted a booster session either in person or by telephone for intervention participants. During this contact, educators focused on the client's progress toward or barriers to meeting specific risk reduction steps and adopting consistent, effective contraceptive and condom use. We selected two months as the time for the booster session and first follow-up to allow participants ample time to make a behavior change; a longer interval may have allowed them to forget the counseling messages or become discouraged. No other booster sessions were conducted, and the data collected at eight and 12 months allowed us to evaluate the longterm effect of the enrollment and booster sessions.

\section{Outcome Measures}

At baseline and at each follow-up, participants were asked about all contraceptive methods used and, for each method, their consistency of use in the last 30 days and their intended use in the next 30 days. If a woman used multiple methods, her most effective method, as determined from an established hierarchy of effectiveness, ${ }^{40}$ was considered her primary method. Women who used condoms were asked about the consistency of use over the last 30 days (every time they had sex, almost every time, sometimes and almost never).

Level of contraceptive use was classified as high, low and none. The high level denoted that a woman took an oral contraceptive every day, consistently used the patch or vaginal ring, used condoms every time or used depot medroxyprogesterone acetate. The low level indicated that she missed oral contraceptives; used the patch, vaginal ring or male condom inconsistently; or used the diaphragm, spermicides, withdrawal, emergency contraception or methods based on fertility awareness. Women who reported no contraceptive use were assigned to the nonuse level.

The primary outcome was change in the level of women's contraceptive use. If a woman increased the level of her contraceptive use (i.e., a nonuser shifted to either a low or high level, or a woman at the low level shifted to the high level), or if she maintained use at a high level, she was considered to have improved her use or maintained the highest level of use. If a woman decreased the level of her contraceptive use (i.e., shifted from the high level to the low level, or from the low level to nonuse), or if she maintained a low level or nonuse, she was not considered to have improved or maintained the highest level of contraceptive use. For women who reported condom use, we assessed whether use was consistent (every time over the last 30 days).

Another measure was women's reported barriers to a high level of use. At baseline and at each follow-up, participants were asked whether these or other situations made it harder for them to use birth control: use of drugs or alcohol, being too sexually aroused, partner's getting angry, too expensive to use, too difficult to obtain, too messy to use, too busy to use, too nervous to use, religious reasons, health problems or side effects caused by birth control.

At the eight-month follow-up, intervention participants were asked about their level of satisfaction with WRAP and the health educators-whether it had been helpful to talk to a counselor about contraception, whether the counselor had focused on their issues and whether the counselor had addressed all of their questions. The five potential responses ranged from strongly agree to strongly disagree.

Unintended pregnancy and chlamydia infection were assessed for all participants over the 12-month study period. Pregnancy and chlamydia tests were conducted at enrollment and at the 12-month completion visit. At each survey, women were asked whether they had had a positive pregnancy test or an STD since the last survey. Medical charts were reviewed for each participant to assess documentation of a pregnancy or diagnosis or treatment of an STD during the study.

\section{Statistical Analyses}

We examined participants' baseline characteristics, both overall and by study group. The level of contraceptive use was measured at baseline and at each follow-up. 
Chi-square tests were used to compare differences in high level of use and improvements in use between the intervention and control groups at each survey. We repeated these tests after stratifying by various sample characteristics to examine effect modification terms. Finally, we used McNemar's chi-square test to assess high level of use and improvement in use within the intervention and control groups.

The sample size was intended to be large enough to measure improvements in the level of women's contraceptive use over time. From our collective clinical and research experience, we anticipated that the proportion of women who improved use or maintained a high level of use would increase by $25 \%$ in the intervention group and by $10 \%$ in the control group over the 12 -month period. With a power of $90 \%$ ( $p=.05$, two-sided), 146 women were required in each study group. In addition, we planned to recruit enough women to assess the occurrence of unintended pregnancy during the study. To calculate the sample size needed for detecting potential differences, we estimated rates of unintended pregnancy of $5 \%$ in the intervention arm and $10 \%$ in the control arm. At 95\% power, we predicted we would need 948 participants. Anticipating that $10 \%$ of participants would be lost to follow-up over the study period, we set a minimum target recruitment of 1,050 participants.

\section{RESULTS}

\section{Participant Characteristics}

From March 2003 to September 2004, a total of 4,101 women were screened for enrollment in the study. Of these women, 1,066 refused, 2,034 were not eligible, and 237 were eligible but either were not interested or did not have sufficient time to participate. Thus, 764 women were eligible and interested, and provided informed consent; 380 were randomly assigned to the intervention group, and 384 to the control group. Of these participants, 85\% completed the two-month follow-up, 91\% completed the eight-month follow-up and $87 \%$ completed the 12-month follow-up; analysis was limited to 737 participants for whom complete follow-up data were collected. We did not obtain an adequate sample size to detect differences between the intervention and control groups for unintended pregnancy and chlamydia infection, so we examined these as secondary outcomes.

Nearly six in 10 participants were between 26 and 44 years old, and the remainder were aged 16-25 (Table 1). Thirty-two percent were currently married, 24\% were formerly married and $45 \%$ had never married. Eightyfour percent had at least a high school education; $62 \%$ were white, $27 \%$ black and $10 \%$ of another race or ethnicity. Participants reported different levels of pregnancy intention: Sixty-four percent said they did not want to get pregnant in the near future, $15 \%$ said they never wanted to get pregnant and $21 \%$ did not know. Seven in 10 women reported having engaged in sexual intercourse in the 30 days prior to enrolling in the study.

\begin{tabular}{|c|c|c|c|}
\hline Characteristic & $\begin{array}{l}\text { Total } \\
(\mathrm{N}=737)\end{array}$ & $\begin{array}{l}\text { Intervention } \\
(\mathrm{N}=365)\end{array}$ & $\begin{array}{l}\text { Control } \\
(\mathrm{N}=372)\end{array}$ \\
\hline \multicolumn{4}{|l|}{ Age } \\
\hline $16-25$ & 41 & 40 & 41 \\
\hline $26-44$ & 59 & 60 & 59 \\
\hline \multicolumn{4}{|l|}{ Marital status } \\
\hline Currently married & 32 & 34 & 30 \\
\hline Formerly married & 24 & 20 & 27 \\
\hline Never-married & 45 & 46 & 43 \\
\hline \multicolumn{4}{|l|}{ Education } \\
\hline$<12$ th grade & 16 & 17 & 15 \\
\hline$\geq 12$ th grade/GED & 84 & 83 & 85 \\
\hline \multicolumn{4}{|l|}{ Race/ethnicity } \\
\hline White & 62 & 65 & 60 \\
\hline Black & 27 & 27 & 28 \\
\hline Other & 10 & 8 & 12 \\
\hline \multicolumn{4}{|l|}{ Pregnancy intention } \\
\hline Not in near future & 64 & 64 & 64 \\
\hline Not ever & 15 & 17 & 14 \\
\hline Do not know & 21 & 19 & 22 \\
\hline \multicolumn{4}{|c|}{ Had had sexual intercourse in last 30 days } \\
\hline Yes & 70 & 71 & 69 \\
\hline No & 30 & 29 & 31 \\
\hline \multicolumn{4}{|l|}{ Type of contraceptive* } \\
\hline Oral contraceptive & 37 & 35 & 38 \\
\hline Condom & 36 & 36 & 36 \\
\hline Injectable/implant & 12 & 12 & 11 \\
\hline Patch/vaginal ring & 6 & 6 & 6 \\
\hline Diaphragm/spermicide & 3 & 2 & 3 \\
\hline Other & 16 & 17 & 14 \\
\hline None & 22 & 20 & 24 \\
\hline \multicolumn{4}{|c|}{ Level of contraceptive uset } \\
\hline High & 59 & 59 & 58 \\
\hline Low & 19 & 21 & 18 \\
\hline None & 22 & 20 & 24 \\
\hline \multicolumn{4}{|c|}{ Barriers to using contraceptives } \\
\hline Yes & 57 & 56 & 57 \\
\hline No & 43 & 44 & 43 \\
\hline Total & 100 & 100 & 100 \\
\hline
\end{tabular}

*Multiple responses allowed. +Based on the method's effectiveness and the consistency of use; see page 23. Note: Percentages may not total $100 \%$ because of rounding.

At enrollment, the most commonly used methods of contraception were oral contraceptives (37\%) and condoms (36\%). Among women who had had sexual intercourse in the 30 days prior to enrollment, consistent use was reported by $94 \%$ of patch or vaginal ring users, $82 \%$ of oral contraceptive users, $56 \%$ of condom users, $33 \%$ of diaphragm or spermicide users, and $50 \%$ of those using other methods (not shown).

In the baseline survey, 59\% of women reported a high level of contraceptive use, and 19\% reported a low level of use; $22 \%$ used no contraceptives. Among women reporting sexual intercourse in the 30 days before baseline, the proportion reporting no use was $5 \%$, and the proportion reporting condom use was 49\% (not shown).

At baseline, 57\% of all participants reported barriers to contraceptive use; the five most common barriers (not 
shown) were forgetting to use (28\%), side effects (19\%), being too sexually aroused (15\%), alcohol use (13\%) and partner opposition (12\%). Overall, women in the intervention and control groups were similar in their social and demographic characteristics, as well as their sexual behavior and contraceptive use.

At the conclusion of the enrollment counseling session, intervention participants selected one or more of the following risk reduction steps: continue current method (214), increase consistency of use (37), start or restart a method (91), obtain medical follow-up (59) or think about starting a method (132).

\section{Intervention Effects}

At the two-month follow-up, participants were asked to report their success in completing their selected risk reduction steps. Ninety percent reported success in continuing their current method, 66\% in increasing their consistency of use, $75 \%$ in starting or restarting a method, $47 \%$ in obtaining medical follow-up for a method and $81 \%$ in thinking about starting a new method.

At the time of enrollment, the proportion of women in the intervention group reporting a high level of contraceptive use was 59\%. Two months later, the proportion who had either improved their level of use or maintained a high level was $72 \%(\mathrm{p}<.001-$ Figure 1$)$. In the control group, the proportions were $58 \%$ and $66 \%$, respectively $(p<.05)$. However, the proportions at two months were not significantly different between the groups. The proportions of intervention and control participants who improved their level of contraceptive use or maintained a high level decreased at eight months to $63 \%$ and $62 \%$, respectively; again, the difference was not statistically significant. At the 12-month follow-up, 64\% of intervention participants reported improvement or an ongoing high level of contraceptive use, compared with $60 \%$ of control participants; this difference was also not statistically significant.

Similarly, among participants who used condoms, the proportion reporting consistent use did not differ between the intervention and control groups at any point in the study, and this proportion did not change significantly over the study period (not shown).

A significantly higher proportion of black women in the intervention group than in the control group reported improvement of contraceptive use or maintenance of a high level of use at the two-month follow-up (72\% vs. $55 \% ; \mathrm{p}<.05)$. A significant difference remained at the 12 month follow-up (60\% vs. $54 \%$ ), although the proportion for the intervention group declined over time. There was no significant difference between proportions of intervention and control participants aged 16-25 years who reported improvement or a high level of use at the twomonth follow-up ( $80 \%$ vs. $67 \%$ ). No differences or trends were found in contraceptive use when participants were analyzed by marital status, education level or pregnancy intention. FIGURE 1. Percentage of women maintaining a high level or
improving their level of contraceptive use from baseline to
12-month follow-up, by study group

$\%$

100
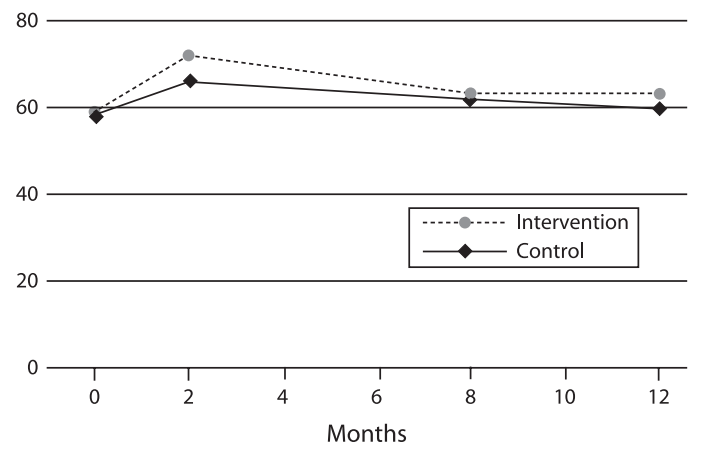

Among women reporting no barriers to contraceptive use, the proportion who improved or maintained a high level of use during the first two months of the study was significantly higher in the intervention than in the control group ( $84 \%$ vs. $73 \%$; $\mathrm{p}=.01$-Figure 2 ). The proportions did not differ significantly at eight or 12 months. Among women who reported barriers to contraceptive use, there were negligible differences at all time points between the proportions of intervention and control participants who improved or maintained a high level of use (Figure 3, page 26).

During the 12-month study, $10 \%$ of participants became pregnant, $1 \%$ received a chlamydia diagnosis and $8 \%$ had another STD diagnosed, according to their questionnaire responses or chart review (not shown); there were no significant differences between intervention and control groups.

Intervention participants reported a high level of satisfaction with WRAP and the health educators. Large proportions strongly agreed or agreed that it had been

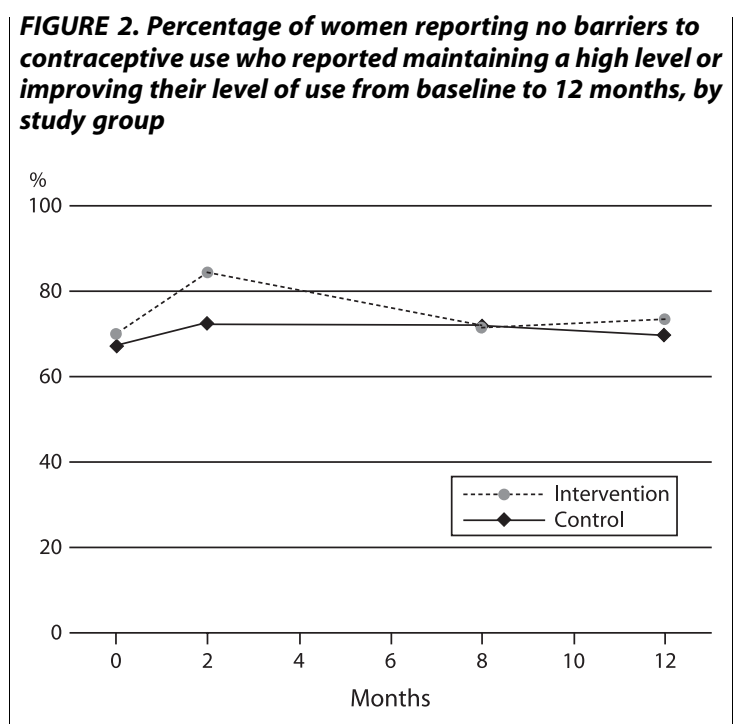




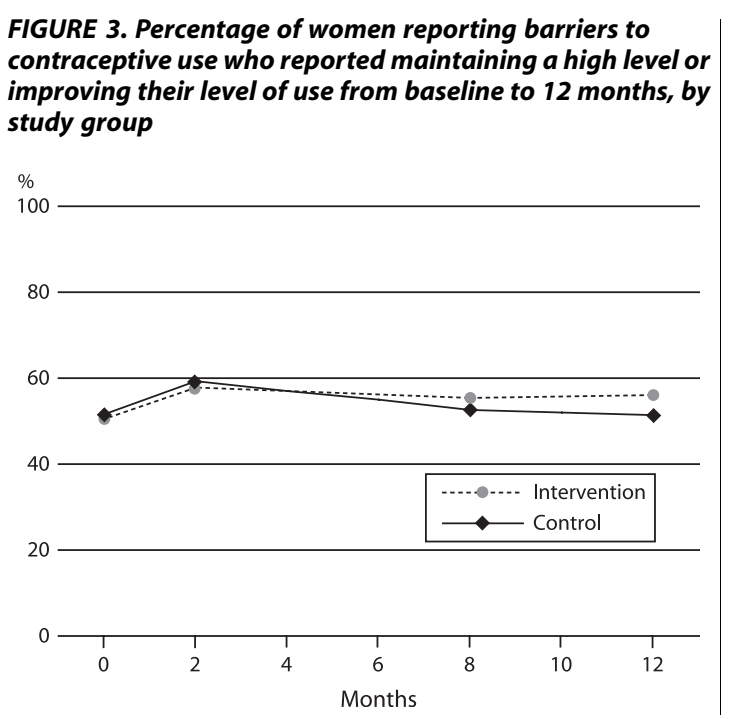

helpful to talk to the educator about contraception (82\%), that the educator had focused on their individual concerns regarding contraception (90\%) and that the educator had addressed all of their questions adequately (93\%).

\section{DISCUSSION}

Despite significant increases in the proportions of intervention and control participants reporting improvement in or maintenance of a high level of contraceptive use at the two-month follow-up, we found no long-term effects of the intervention or significant differences between the two groups. In addition, for women reporting condom use, the intervention did not increase consistency of use.

We propose several implications of our findings. The positive findings at two months and the negative findings at eight and 12 months suggest that repeated counseling or booster sessions may be needed to help women maintain or improve their level of contraceptive use. Other areas of research (e.g., nutrition, ${ }^{41}$ smoking cessation $^{42}$ and physical activity ${ }^{43}$ ) have found the need for repeated counseling to support positive behavior change. The lack of improvement in consistency of condom use may be related to a lower risk, or a lower perceived risk, of STDs among the study participants than we expected.

Many control participants improved their level of contraceptive use or maintained a high level of use during the first two months despite a lack of pregnancy and STD prevention counseling from the WRAP health educators. This may be related to the completion of the baseline questionnaire, which included many questions about contraceptive use and the risk of unintended pregnancy and STDs. Exposure to these questions may have prompted control participants to think more about these issues and to use contraceptives more effectively. However, if this were the primary explanation for our findings, the completion of questionnaires at eight and 12 months might have led women to a continuation of their use of effective contraception over the entire study period, which it did not.
Another potential explanation for the increase in the level of contraceptive use among control participants may have been changes in provider behaviors beyond the control of the WRAP study. This study was not intended to influence or replace provider care in the primary health care settings. However, given the study team's presence in the clinics, providers were aware that patients were being recruited into a randomized controlled trial to evaluate a counseling intervention. Some providers may have been reminded, just by the presence of the WRAP team, to provide counseling for pregnancy and STD prevention.

These findings support earlier research showing that many women are at risk of unintended pregnancy and STDs because of low levels of contraceptive use or nonuse. In this study, having less than a high level of contraceptive use was defined as using a less effective method, or using a method inconsistently. Our findings also help demonstrate the difficulty of defining the concept of unintended pregnancy and the potential for ambivalence, given that $21 \%$ of participants were not sure whether they wanted to become pregnant or not.

Intervention participants reported a high level of satisfaction with the WRAP counseling and the health educators. At weekly team meetings, the educators reported many positive interactions with participants, and the study team received positive feedback from many health care providers in the clinical settings where the study was evaluated. Additionally, both participants and providers appreciated the efforts of health educators in devoting the time to discuss the complex issues of contraceptive use and the prevention of pregnancy and STDs.

\section{Strengths and Limitations}

The strengths of this study include the randomized controlled design, the 12-month follow-up period, the high participant retention rates and the inclusion of behavior-change theory in the design and implementation of the intervention. Limitations include the reliance on self-reporting for contraceptive use and barriers to use. Self-report may result in bias because participants may underreport the amount and level of risk-taking and nonuse or underuse of contraceptives. Participants may also have experienced barriers to contraceptive use that we were unable to identify with a written survey. The lack of power to detect differences in pregnancy and chlamydia rates between the groups was also a concern, given that these outcomes occurred less frequently than we had predicted. Furthermore, despite training and management oversight to standardize the intervention delivery, there may have been counseling inconsistencies among the WRAP health educators. In addition, some possible influences in the clinical settings were beyond the scope of the study (e.g., provider knowledge, practices and attitudes regarding contraceptive and reproductive health counseling). These limitations restrict the conclusions we can draw about the lack of a significant difference in 
contraceptive use between the intervention and control groups, especially after the first two months.

\section{Conclusion}

This study confirms the complexity of addressing women's risk of unintended pregnancy and STDs in clinical settings, particularly the difficulty of assessing risk, the many barriers to contraceptive use, the differences in method effectiveness and the factors that control the consistency with which women use a method. Furthermore, intervention participants may have benefited more if the counseling had been continued in multiple booster sessions. Future research should examine whether repeated counseling enables women to maintain the preventive behaviors needed to reduce their risk of unintended pregnancy and STDs. If such counseling is found to reduce women's sexual and reproductive risks, an economic evaluation of the costs and benefits will help in assessing whether the wider provision of pregnancy and STD prevention counseling can make a significant contribution to improving women's reproductive health.

\section{REFERENCES}

1. Finer LB and Henshaw SK, Disparities in rates of unintended pregnancy in the United States, 1994 and 2001, Perspectives on Sexual and Reproductive Health, 2006, 38(2):90-96.

2. Henshaw SK, Unintended pregnancy in the United States, Family Planning Perspectives, 1998, 30(1):24-29 \& 46.

3. Brown SS and Eisenberg L, eds., The Best Intentions: Unintended Pregnancy and the Well-Being of Children and Families, Washington, DC: National Academy Press, 1995

4. Koonin LM, Smith JC and Ramick M, Abortion surveillanceUnited States, 1991, Morbidity and Mortality Weekly Report, 1995, 44(2):23-53.

5. Kost K, Landry DJ and Darroch JE, The effects of pregnancy planning status on birth outcomes and infant care, Family Planning Perspectives, 1998, 30(5):223-230.

6. Kost K, Landry DJ and Darroch JE, Predicting maternal behaviors during pregnancy: does intention status matter? Family Planning Perspectives, 1998, 30(2):79-88.

7. Centers for Disease Control and Prevention, Physical violence during the 12 months preceding childbirth-Alaska, Maine, Oklahoma, and West Virginia, 1990-1991, Morbidity and Mortality Weekly Report, 1994, 43(8):132-137.

8. Weinstock H, Berman S and Cates W, Jr., Sexually transmitted diseases among American youth: incidence and prevalence estimates, 2000, Perspectives on Sexual and Reproductive Health, 2004, 36(1):6-10.

9. Chandra A and Stephen EH, Impaired fecundity in the United States: 1982-1995, Family Planning Perspectives, 1998, 30(1): 34-42.

10. Petersen $\mathrm{R}$ et al., How contraceptive use patterns differ by pregnancy intention: implications for counseling, Women's Health Issues, 2001, 11(5):427-435.

11. Chandra A et al., Fertility, family planning, and reproductive health of U.S. women: data from the 2002 National Survey of Family Growth, Vital and Health Statistics, 2005, Series 23, No. 25.

12. Mosher WD et al., Use of contraception and use of family planning services in the United States: 1982-2002, Advance Data from Vital and Health Statistics, 2004, No. 350.
13. Abma JC et al., Teenagers in the United States: sexual activity, contraceptive use, and childbearing, 2002, Vital and Health Statistics, 2004, Series 23, No. 24.

14. Office of the Surgeon General, The Surgeon General's Call to Action to Promote Sexual Health and Responsible Sexual Behavior, Washington, DC: U.S. Department of Health and Human Services (DHHS), 2001

15. DHHS, Healthy People 2010, Washington, DC: DHHS, 2000.

16. U.S. Preventive Services Task Force, Guide to Clinical Preventive Services, second ed., Baltimore: Williams \& Wilkins, 1996.

17. Trussell J, Contraceptive failure in the United States, Contraception, 2004, 70(2):89-96.

18. Ewing GB et al., Self-report of delivery of clinical preventive services by U.S. physicians: comparing specialty, gender, age, setting of practice, and area of practice, American Journal of Preventive Medicine, 1999, 17(1):62-72.

19. Stange KC et al., Direct observation of rates of preventive service delivery in community family practice, Preventive Medicine, 2000, 31(2):167-176

20. Weisman CS et al., Contraceptive counseling in managed care: preventing unintended pregnancy in adults, Women's Health Issues, 2002, 12(2):79-95

21. Lewis CE, Sexual practices: are physicians addressing the issues? Journal of General Internal Medicine, 1990, 5(Suppl. 5):78-81.

22. Merrill JM, Laux LF and Thornby JI, Why doctors have difficulty with sex histories, Southern Medical Journal, 1990, 83(6): 613-617.

23. Makadon HJ and Silin JG, Prevention of HIV infection in primary care: current practices, future possibilities, Annals of Internal Medicine, 1995, 123(9):715-719.

24. Boekeloo BO et al., Frequency and thoroughness of STD/HIV risk assessment by physicians in a high-risk metropolitan area, American Journal of Public Health, 1991, 81(12):1645-1648.

25. Haley $\mathrm{N}$ et al., Sexual health risk assessment and counseling in primary care: how involved are general practitioners and obstetriciangynecologists? American Journal of Public Health, 1999, 89(6): 899-902

26. Moos MK, Bartholomew NE and Lohr KN, Counseling in the clinical setting to prevent unintended pregnancy: an evidence-based research agenda, Contraception, 2003, 67(2):115-132.

27. Cabral RJ et al., Paraprofessional delivery of a theory based HIV prevention counseling intervention for women, Public Health Reports, 1996, 111(Suppl. 1):75-82.

28. Terry MA et al., The Women and Infants Demonstration Project: an integrated approach to AIDS prevention and research, AIDS Education and Prevention, 1999, 11(2):107-121.

29. St. Lawrence J et al., HIV risk reduction for incarcerated women: a comparison of brief interventions based on two theoretical models, Journal of Consulting and Clinical Psychology, 1997, 65(3):504-509

30. Kelly JA et al., The effects of HIV/AIDS intervention groups for high-risk women in urban clinics, American Journal of Public Health, 1994, 84(12):1918-1922.

31. Carey MP et al., Enhancing motivation to reduce the risk of HIV infection for economically disadvantaged urban women, Journal of Consulting and Clinical Psychology, 1997, 65(4):531-541.

32. Shain RN et al., A randomized, controlled trial of a behavioral intervention to prevent sexually transmitted disease among minority women, New England Journal of Medicine, 1999, 340(2):93-100.

33. Miller WR and Rollnick S, Motivational Interviewing: Preparing People to Change Addictive Behavior, New York: Guilford Press, 1991.

34. Britt E, Hudson SM and Blampied NM, Motivational interviewing in health settings: a review, Patient Education and Counseling, 2004, 53(2):147-155. 
35. Petersen R et al., Applying motivational interviewing to contraceptive counseling: ESP for clinicians, Contraception, 2004, 69(3): 213-217.

36. Bandura A, Self-efficacy: toward a unifying theory of behavioral change, Psychological Review, 1977, 84(2):191-215.

37. Prochaska JO and DiClemente CC, The Transtheoretical Approach: Crossing Traditional Boundaries of Therapy, Homewood, IL: DowJones/Irwin, 1984.

38. Jobe J and Mingay D, Cognition and survey measurement: history and overview, Applied Cognitive Psychology, 1991, 5(3):175-192.

39. Institute of Medicine, Crossing the Quality Chasm: A New Health System for the 21st Century, Washington, DC: National Academy Press, 2001.

40. Hatcher RA et al., Contraceptive Technology, 18th ed., New York: Ardent Media, 2004.

41. Pignone MP et al., Counseling to promote a healthy diet in adults: a summary of the evidence for the U.S. Preventive Services
Task Force, American Journal of Preventive Medicine, 2003, 24(1): 75-92.

42. Manfredi $C$ et al., Evaluation of a motivational smoking cessation intervention for women in public health clinics, Preventive Medicine, 1999, 28(1):51-60.

43. Simons-Morton DG et al., Effects of interventions in health care settings on physical activity or cardiorespiratory fitness, American Journal of Preventive Medicine, 1998, 15(4):413-430.

\section{Acknowledgments}

Funding for this work came from the Centers for Disease Control and Prevention/Association of Teachers of Preventive Medicine cooperative agreement TS-0768. The conclusions and opinions expressed here are those of the authors and not necessarily those of the funder.

Author contact: ruth_petersen@unc.edu 